\title{
Management of self-harm injuries in the maxillofacial region: a report of two cases and review of the literature.
}

\section{ABSTRACT}

Clinicians face numerous challenges when managing psychiatric patients who self-inflict injuries within the maxillofacial region. In addition to a complex clinical examination, there are both surgical and psychiatric factors to consider, such as the risk of damaging vital structures, the exacerbation of their psychiatric status, and the long-term psychosocial and aesthetic sequelae. Two cases of adolescents who repeatedly self-inflicted wounds and/or inserted foreign bodies (FBs) into the face, scalp and neck are reported herein. The different treatment modalities were based on full evaluation of the patient's clinical, medical and diagnostic tests coupled with a psychiatric assessment. The decision for conservative management or surgical intervention was made according to the presence and location of the FBs, the degree of haemorrhage, signs and symptoms of infection, and unpleasant scars which could lead to long-term psychological impairment. In most cases, FBs were removed, and the wounds were toileted and closed under local or general anaesthesia. We advocate a holistic approach via a multi-disciplinary team, which is deemed essential to provide the highest quality of care for patients to reduce the risk of further relapses. Lastly, a satisfactory aesthetic outcome is always paramount in order to achieve long-term psychological and physical welfare.

\section{INTRODUCTION}

Self harm is defined as the deliberate, direct destruction or alteration of body tissue resulting in injury severe enough for tissue damage to occur. ${ }^{1}$ It primarily constitutes an emotion regulation strategy and/or form of selfpunishment, which serves to temporarily alleviate psychological distress, at the cost of physical injury and longterm impairment of psychological and physical welfare. ${ }^{2,3}$ It usually starts in adolescence, and covers a broad spectrum of behaviours where the injury is self-inflicted. ${ }^{2,4}$ Although self-harm can occur within the whole population, it is more prevalent among those who are socially deprived and/or have a history of major childhood trauma (emotional, physical or sexual abuse), ${ }^{5,6}$ The most common method of self-injury is by cutting oneself; others range from minor lesions such as burning, self-poisoning and alcohol abuse, to serious permanent 
damage including eye enucleation, genital mutilation and amputation of the tongue or ear. 2,4,7 Although suicide is not the intention of self-harm, the relationship between self-harm and suicide is often complex; as self-harming behaviour may be potentially life-threatening. Furthermore, there is also an increased risk of suicide in individuals who self-harm ${ }^{5,8,9,10}$, especially in males with previous psychiatric history and/or self-harm. ${ }^{11}$ According to data collected by Owens et al, $1.8 \%$ of people who harm themselves commit suicide within a year following an incident ${ }^{9}$ and those who repeat self-harm are twice as likely to die by suicide compared with those who selfharm only once ${ }^{10}$. Individuals who self-harm are also at an increased risk of premature death from natural causes, usually caused by circulatory or digestive illness. They die on average 30 years earlier than the norm; the mean age of death for women who self-harm is 54 and for men, $50 .{ }^{5}$

Self-harm imposes a significant cost to society; besides increased health care costs as a result of multiple presentations to health professionals, there can be an effect on workplace attendance, the costs of long-term disability and premature mortality. 5,10 The last few years have seen a rise in the number of self-harm cases reported in the relevant literature ${ }^{12}$, although only a handful have been described within the maxillofacial specialty. Figures published in 2014 suggested a 70\% increase in 10-14 year olds attending to the accident and emergency department (A \& E) for self-harm related reasons over the preceding 2 years. ${ }^{13}$ It is therefore essential that clinicians learn to recognize cases which could potentially involve severe outcomes. ${ }^{5}$ Lewis and colleagues estimated that additional interventions following self-harm reduces the rate of subsequent suicide by $25 \% .^{9}$

We present two cases of adolescents who attended $A \& E$ on multiple occasions due to repeated episodes of self-harming behaviour; mainly the insertion of FBs and self-inflicted cuts to the face, neck and scalp.

The aims of this report are to identify suitable methods of managing patients that perform self-harming behaviour in the maxillofacial region by taking into account their overall psychiatric condition as well as the nature and severity of the injuries. We also intend to educate the OMS Community about the increasing problem of selfinjury and the importance of its identification followed by an appropriate psychiatric referral to avoid fatal outcomes.

\section{CASE REPORTS}




\section{Case 1}

A 17-year old female presented in A \& E with self-harm injuries to the head and neck region on eight separate occasions over a two-year period (see table 1). The patient had a history of depression, post-traumatic stress disorder (PTSD), self-harming behaviour, and was diagnosed with emotional instability and personality disorder. Her regular medication included: olanzapine $15 \mathrm{mg}$ daily, hyoscine patch $1 \mathrm{mg} / 72 \mathrm{~h}$, promethazine $50 \mathrm{mg}$ daily, sertraline $50 \mathrm{mg}$ daily, colecalciferol 400 units daily, oxytetracycline $500 \mathrm{mg}$ four times a day, and multivitamins one tablet daily. She was a long-standing inpatient in one of the largest mental health hospitals in the U.K.

The patient first presented to $A \& E$ in February 2012 with a $6 \mathrm{~cm}$ incised wound to the right cheek caused by a shard of glass, and then 17 months later with puncture wounds to the left cheek caused by the insertion of a hairgrip and a pen refill cartridge (see image 1). Following these episodes, the patient was then transferred to a medium security unit as a result of the deterioration in her mental state.

The patient presented again in August 2013 and on two further occasions later that year with profuse bleeding from the ventral surface of the tongue caused by a ceramic tile shard, a sharp piece of plastic and a shard of glass respectively, whereby each object had been inserted into the tongue. The wounds were managed with FB removal, haemorrhage control, and toilet and closure under local anaesthesia.

A further attendance involved symptoms of oral pain, dysphonia and dysphagia following the insertion of a straight paper clip into the tongue. A soft tissue radiograph confirmed the paperclip was fully buried and incidentally a nail in the neck with its head in close proximity to the trachea (see image 2). No surgical intervention was performed at that stage although relief of symptoms and a course of antibiotics were provided to prevent secondary infection.

Further episodes included profuse sublingual bleeding caused by the insertion of a clothing zip, and a laceration to the right cheek extending up to the temple caused by a gel pen. Examination showed the parotid duct was intact, and on both occasions the facial nerve was fully functional. Management consisted of removal of the FBs, debridement and closure under local anaesthesia.

\section{Case 2}


A 23-year old female presented in A \& E on forty-eight separate occasions with self-inflicted injuries to multiple sites on the body over a two year period (see table 1). Fourteen of the episodes involved injuries to the head and neck region and a total of fourteen imaging techniques including CT and MRI scans, facial bones, and panoral views were taken. The patient had borderline type, emotionally unstable personality disorder, severe akathisia, iron-deficiency anaemia and recurrent self-harming behaviour. Her regular medication included: quetiapine 300 $\mathrm{mg}$ daily, propranolol $40 \mathrm{mg}$ daily, sodium valproate $600 \mathrm{mg}$ daily, sodium feredetate $380 \mathrm{mg} 3$ times daily, clonazepam $10 \mathrm{mg}$ daily, and chlorpromazine $75 \mathrm{mg}$ daily. She was also an inpatient in the aforementioned psychiatric hospital.

The majority of injuries involved the insertion of FBs into the forehead and scalp, of which some of the objects isolated were: a pencil, coins, a clothing zip, a paper clip, and a piece of chain (see images 3-4). Most of the injuries were managed with removal of the FBs and debridement and closure of the wounds under general anaesthesia. On one occasion the patient declined surgical treatment. Despite this, a decision was made in the patient's best interest and surgical intervention was provided under general anaesthesia.

Her mental health recovery within the Psychiatric Unit was a long and complex process involving both pharmacological and psychological treatment; it consisted of stabilising her mental health condition, management of substance abuse and identifying the reasons that triggered her self-injuries habits. The patient learnt how to control stressful thoughts or feelings through coping mechanisms, as well as how to ask for emergency counselling when in danger of recurrence. Fourteen months after the last self-harm episode, the patient was considered psychiatrically stable and was discharged home to be closely supervised by the Community Mental Health Services. However, the patient's recovery in the community was deemed at risk as a result of the aesthetic consequences of her self-harm injuries, in particular a noticeable scar on the forehead adjacent to an area of alopecia caused by repetitive injury. The patient was therefore referred by her psychiatrist to plastics for scar revision surgery, for which a satisfactory aesthetic result was accomplished. To this date the patient remains in the community and no further self-harm episodes have been registered.

\section{DISCUSSION}


Of the cases presented, for the majority of penetrating self-harm injuries in the head and neck region, the preferred management was surgical treatment. However, on some occasions, the wounds were treated conservatively and no surgical treatment was provided because either there was a deterioration of the patient's mental state leading to an increased number of self-harm episodes, or the risk of damage to vital structures was extremely high.

Of the cases reported here, all the vital structures were found to be fully functional following clinical examination and diagnostic imaging, and no sequelae were encountered post-operatively According to Tabariary et al, factors to consider when treating a penetrating facial injury include: location and extent of the object, type of FB, vital structures in its proximity, possible sequelae of removing the FB, general extent of the injury to the hard and soft tissues, and the patient's general medical condition. ${ }^{14}$ Apart from the location of a FB, a penetrating injury to the face can also damage deeper anatomical structures such as the facial nerve, the salivary glands and greater and lesser arteries and veins. Therefore, mandatory neck exploration is advocated for patients with penetrating neck trauma by certain surgeons because of the risk of damage to significant anatomical structures ${ }^{15}$ Although the facial nerve is completely embedded in soft tissue, traumatic injuries to it account for up to $5 \%$ of all cases of facial nerve disorders. Sialoceles and parotid fistulae are the most common post-traumatic complications of an unrecognised parotid duct injury. ${ }^{16,17}$

The implantation of FBs may be accidental, iatrogenic, or in certain situations deliberate as described in this case report. ${ }^{18}$ Several authors have reported that approximately one-third of all FBs are initially missed and on many occasions are an incidental finding on radiographs ${ }^{19,20}$ The diagnosis of a FB within the maxillofacial region can prove challenging, particularly if access is difficult, the object is very small and/or is in close anatomical relation to vital structures. ${ }^{19,21}$ Determining the location of a FB before its removal is essential as blind exploration can cause further trauma to the tissue and displace the foreign body into deeper fascial planes. ${ }^{22}$ Sharp FBs can also migrate over time towards vital structures that subsequently could cause severe complications. ${ }^{21}$ In these two cases, there was no migration of the FBs into deeper fascial planes.

Of the cases reported, plain radiographs were taken in $90 \%$ of attendances to identify and determine the location of the FBs. This is in keeping with other studies, where conventional radiographs were usually the first line among imaging techniques due to their low cost and effectiveness at diagnosing metal objects. ${ }^{14}$ There are many 
different imaging techniques available to detect the location of FBs, in particular, CT scans are mainly used to identify metal FBs in close proximity to vital structures due to their ability to determine the extension of the injury and the precise location of the object. In the case of our second patient, the surgical team did not organise a CT scan for the nail in the cervical region as clinically there was no concern. Despite the benefits of MRI scans and ultrasound investigations in detecting penetrating wood injuries that are not obstructed by bone or gas, MRI scans need to be avoided when there is suspicion of a metallic foreign body located completely internally due to the danger caused by the movement of the metallic object. ${ }^{14,19}$

The nail in the neck found as an incidental finding in case one was managed conservatively because it was not impinging on the trachea, there was no impairment of mechanical function and no signs or symptoms of infection. Furthermore based on the evidence above, there was no indication to remove the nail at the time of its discovery due of the following reasons: the potential damage to vital structures with exploration of the region was extremely high, the patient's mental health had deteriorated at that time, and the risk of repeated general anaesthesia was also high. The patient was provided with supportive care, management of the symptoms and prophylactic antibiotics. Besides, she was kept under regular review to monitor the location of the FB. In contrast, the FBs reinserted into the scalp wound for case two were managed in the patient's best interest with surgical intervention, because of the high risk of sepsis and irritation to the soft tissues. This approach is in keeping with the following indications stated in the literature for the removal of FBs from soft tissue: reactivity, heavy contamination, toxicity and impingement of vital structures, impairment of mechanical function, intra-auricular location, intravascular location, persistent pain, established infection, allergic reaction, cosmetics and psychological distress. ${ }^{23}$ Occasionally, the removal of small foreign body particles may not be possible, but larger foreign bodies should always be removed because they may cause pain, tenderness or deformity. ${ }^{22}$ Whilst some foreign bodies may be left in situ for good clinical reasons, most are removed prior to the onset of complications, notably infection. ${ }^{24}$

Local anaesthesia was the preferred treatment method in case one; however, the patient in case two underwent general anaesthesia on more than eight occasions. This was due to the size, severity and location of wounds, the deterioration of the patient's mental state and because on the majority of the occasions, the patient declined 
local anaesthesia. FBs in the maxillofacial region are usually best managed under general anaesthesia, although local anaesthesia often is satisfactory if the imaging confirms a superficial location. ${ }^{14,15,19}$

The repeated episodes of trauma to the chronically infected scalp wound without the insertion of FBs in case two were managed conservatively with a course of antibiotics and delayed wound care. This treatment approach is indicated in the management of contaminated, dirty wounds, because it enables a healthy bed of granulation tissue to form and also decreases the bacterial load in the wound..$^{25}$

In both cases presented, the patients were known as recurrent self-harmers. However it is extremely important in our specialty to identify them as nearly two-thirds of adolescents who self-cut in other parts of the body other than the arms have suicidal thoughts. ${ }^{12}$ The responsible surgeon has to establish the patient's role in their injury through a comprehensive history of events; contradictions in the patient's story, injury not consistent with the explanation given, previous psychiatric history and substance abuse are signs of alert to suspect self-injury. ${ }^{5}$ As it is demonstrated in both of our cases, previous self-harm injuries as well as co-existent mental health conditions, particularly personality disorder and schizophrenia, are the strongest predictors for recurrent selfharm. 10,11 Emergency departments and local mental health services have a duty to jointly plan effective liaison psychiatric services that are available 24 hours a day..$^{13}$ All patients who present with self-harm injuries should have a psycho-social risk assessment carried out by a mental health professional. ${ }^{5}$ This includes: an assessment of the patient's mental capacity, level of distress and possible mental illness, development of an emergency care plan, and referral to the most adequate team. ${ }^{5,13}$ If the patient wishes to leave before this has been undertaken, an assessment of the mental capacity and identification of possible mental illness needs to be carried out. This assessment needs to be recorded in the clinical notes and immediately passed on to the patient's GMP and other relevant mental health services to enable rapid follow-up. ${ }^{26}$ However if the patient's capacity diminishes and/or the presence of a significant mental illness is established, an urgent mental health referral is required. ${ }^{13}$ Recent studies have demonstrated that only between $4-20 \%$ of adolescents who present in A\&E with self-injuries received an appropriate psychiatric follow up. ${ }^{26}$ This is the why, in the case of children and young adults who self-harm, it is highly recommended to establish whether they are already involved with the child and adolescent mental health services (CAMHS), and if not, an appropriate referral to the service should be made. ${ }^{13}$ In both our cases, they were in-patients at a Psychiatric Unit and therefore their psychosocial and risk 
assessment was carried out before their visit to A\&E. They attended with their mental health workers and a care plan was made after the treatment of their injuries with the input of the surgical team.

Due to the severity of mental health of both patients presented, their psychiatric treatment was delivered within a Hospital; however when the risk is low and the patient expresses preference, the treatment can be managed within the Community Mental Health Team. ${ }^{27}$ In most cases, psychological treatment is recommended for people who self-harm. Pharmacological treatment will be needed if, like in the case of both our patients, mental illness is present. Diagnoses and treatment of the root cause of self-harm is thought to be the best approach when treating. Psychodynamic therapy, cognitive behavioural therapy (CBT), family intervention and interpersonal therapy appear to be effective on the treatment of self-harm behaviour, particularly in children and adolescents. ${ }^{28,29}$ CBT aims to identify the negative emotions and problem-solving deficit linked to self-ham, in order to develop behavioural abilities to cope with all the situations that initiate self-harm. Psychodynamic therapy emphases on the effect of past experiences into their actual feelings and thoughts. ${ }^{30} \mathrm{In}$ the case of our second case, she was treated with a modified CBT: dialectical behavioural therapy (DBT); a technique designed for Borderline Personality Disorder. A successful behavioural method consists of generating an alternative behaviour instead of self-harm; this might involve the use of safer self-harm method like snapping a rubber band on the wrist or the removal of objects used for self-injury from easy reach. ${ }^{11}$ Self-harm patients treated within the community should have an emergency counselling contact in case the urge to self-harm arises. ${ }^{32}$ Repetitions of self-harm is common, particularly in the first weeks after the previous presentation as both our cases demonstrate..$^{5,10}$ On average, $16 \%$ of self-harm patients repeat self-harm within one year. ${ }^{10}$ The risk of subsequent suicide after self-injury is highest within the first 6 months, although it has been shown to remain increased for a long period of time, perhaps even for a lifetime. ${ }^{6}$

\section{CONCLUSION}

The management of the self-harm injuries within the maxillofacial region can be highly demanding, thus a detailed history, exhaustive clinical examination and appropriate diagnostic imaging should be performed for every patient. We recommend a structured and cohesive multidisciplinary team approach as the key in achieving the best outcome for these patients, from both a psychiatric and surgical point of view to reduce the risk of further relapses. 


\section{ACKNOWLEDGMENTS}

The authors would like to thank Mr James Gallagher, Consultant in Oral and Maxillofacial Surgery, for his work and sharing information regarding this study.

\section{REFERENCES}

1. Gratz, KL. Risk factors for and functions of deliberate self-harm. An empirical and conceptual review. Clin Psychol (New York) 2003;10:192-205

2. Mikolajczak M, Petrides KV, Hurry J. Adolescents choosing self-harm as an emotion regulation strategy: the protective role of trait emotional intelligence. Br J Clin Psychol 2009;48:181-93

3. Chapman AL, Gratz KL, Brown MZ. Solving the puzzle of deliberate self-harm: The experimental avoidance model. Behav Res and Ther 2006; 44:371-94

4. Kehan J, Pattison EM. Proposal for a distinctive diagnosis: The deliberate self-harm syndrome(DSH). Suicide Life Treat Behav 1984;14:17-35

5. Kinahan JC, MacHale S. The surgeon and self-harm: at the cutting edge. The Surgeon 2014; 12: 345-349

6. Bilen $\mathrm{K}$, Ottoson $\mathrm{C}$, Castren $\mathrm{M}$ et al. Deliberate self-harm patients in the emergency department: factors associated with repeated self-harm among 1524 patients. Emerg Med J 2011; 28:1019e1025. doi:10.1136/emj.2010.102616

7. Bachman JA. Self-injurious behaviour: a behavioural analysis. J Abnorm Psychol 1972; 80 (3): 211-24

8. Hawton K, Zahl D, Weatherall R. Suicide following deliberate self-harm: Long-term follow-up of patients who presented to a general hospital. Br J Psychiatry 2003;182;537-42.

9. Crawford MJ, Thomas O, Khan N et al. Psychosocial interventions following self-harm. Systematic review of their efficacy in preventing suicide. Br J Psychiatry 2007;190: 11-17

10. Larkin C, Di Blasi C, Arensman E. Risk factors for repetition of self-harm. A systematic review of prospective hospital-based studies. Plos One 2014; Vol 9- Issue 1: e84282 
11. Hawton $\mathrm{K}$, Bergen $\mathrm{H}$, Kapur $\mathrm{N}$ et al. Repetition of self-harm and suicide following self-harm in children and adolescents: findings from the multicentre study of self-harm in England. J Child Psychol Psychiatry 2012; $53: 1212-1219$

12. Laukkanen E, Rissanen ML, Tolmunen T et al. Adolescents self-cutting elsewhere than on the arms reveals more serious psychiatric symptoms. Eur Child Adolesc Psychiatry 2013;22: 501-510

13. NICE (2004) Self-Harm: the Short-Term Physical and Psychological Management and Secondary Prevention of Self-Harm in Primary and Secondary Care. NICE clinical guideline 16. Available at www.nice.org.uk/CG16 [NICE guideline]

14. Tabariai E, Sandhu S, Alexander G et al. Management of facial penetrating injury- A case report. J Oral Maxillofac Surg 2010;68:182-7

15. Biffl WL, Moore EE, Dagmar H et al. Selective management of penetrating neck trauma based on cervical level of injury. The American Journal of Surgery 1997;174: 678-682

16. Li J, Goldberg G, Munin MC et al. Post-traumatic bilateral facial palsy: a case report and literature review. Brain Inj 2004;18(3):315-20

17. Meer M, Siddiqi A, Morkel JA, van Rensburg $P$ et al. Knife inflicted penetrating injuries of the maxillofacial region: a descriptive record-based study. Injury, Int J Care Injured 2010; 41:77-81

18. Quiamud D. Foreign bodies in maxillofacial region. J Pak Dent Assoc 2001;10(3):153-7

19. de Santana TS, Linard RA, Rocha Melo A, Araujo de Moraes HH, Dourado E. Current approach in the management of patients with foreign bodies in the maxillofacial region. J Oral Maxillofac Surg 2011;69:2376-82

20. Rudagi BM, Halli R, Kini Y, Kharkhar V , Saluja H. Foreign bodies in facial trauma: report of 3 cases. J Maxillofac Oral Surg 2013;12(2):210-13

21. Takaoka K, Hashitani S, Toyohara $Y$ et al. Migration of a foreign body (staple) from the oral floor to the submandibular space: case report. Br J Oral Maxillofac Surg 2010;48:145-6 
22. Vikram A, Mowar A, Kumar S. Wooden foreign body embedded in the zygomatic region for 2 years. $J$ Maxillofac Oral Surg 2012;11(1):96-100

23. Fonseca RJ, Walker RV. Oral and maxillofacial trauma. $2^{\text {nd }}$ edn. Philadelphia: W.B Saunders Company. 1997.

24. Cameron N, Phillips B. Facial infection secondary to occult foreign body. Int J Oral Maxillofac Surg 2006;35:373-5

25. Ueeck BA. Penetrating injuries to the face: Delayed versus primary treatment-Consideration for delayed treatment. J Oral Maxillofac Surg 2007;65:1209.

26. Tørmoen AJ, Rossow I, Mork E et al. Contact with chld and adolescent psychiatric services among selfharming and suicidal adolescents in the general population: a cross sectional study. Child Adolesc Psychiatry Ment Health 2014; 8: 13

27. Hume M, Platt S. Appropriate interventions for the prevention and management of self-harm: a qualitative exploration of service-users' views. BMC Public Health 2007; 7:9

28. Glenn CR, Franklin JC, Nock MK. Evidence-based psychosocial treatments for self- injurious thoughts and behaviours in youth. J Clin Child Adolesc 2015; 44 (1): 1-29

29. Ougrin D, Tranah T, Leigh E et al. Practitioner review: Self-harm in adolescents. J Child Psychol Psyc 2012; 53 (4): $337-50$

30. Slee N, Garnefski N, van der Leeden R et al. Cognitive-behavioural intervention for self-harm: randomised controlled trial. Br J Psychiatry 2008; 192, 202-211

31. Klonsky ED, Glenn CR. Resisting Urges to Self-Injure. Behav Cogn Psychoth 2008; 36 (02): 211-220

32. Hawton K, Arensman E, Townsend E et al. Deliberate self-harm: systematic review of efficacy of psychosocial and pharmacological treatments in preventing repetition. Brit Medl J 2008; 317: 441-447 
Image 1: The radiograph shows the insertion of foreign bodies into the left cheek on second A\&E attendance for case report 1.

Image 2: The radiograph shows the paper clip in the tongue and an incidental finding of a nail in neck on sixth A\&E attendance for case report 1.

Image 3: The radiograph shows the insertion of foreign bodies into the left side of forehead on the first A\&E attendance for case report 2.

Images 4: The radiograph shows multiple foreign bodies inserted into the forehead scalp bilaterally on the fifth A\&E attendance for case report 2.

Table 1: Episodes of self-harm 